Cevallos Ammiraglia, D.A. Campo jurídico y campo médico: algunas analogías y diferencias. Derecho y Ciencias Sociales. Noviembre 2020 - Abril 2021. No 24 .Pgs 1 -16 ISSN 1852-2971. Instituto de Cultura Jurídica y Maestría en Sociología Jurídica. Facultad de Ciencias Jurídicas y Sociales. Universidad Nacional de La Plata. Argentina.

\title{
Campo jurídico y campo médico: algunas analogías y diferencias
}

Legal field and medical field: some analogies and differences

Diego A. Cevallos Ammiraglia

\section{Resumen}

El trabajo tiene como objeto analizar puntos de reflexión teóricos desde la perspectiva de Bourdieu en el campo del derecho y en el campo médico.

Pierre Bourdieu, problematizó tangencialmente el campo de la salud, es por ello que creemos necesario un aporte en el estudio del campo sanitario.

En un plano conjetural, ajeno a la investigación empírica, se detectan un mayor número de analogías que diferencias entre ambos espacios sociales. Quizá, dicha situación encuentre una respuesta fundada en la presencia insoslayable del Estado, como "metacampo". Por la historicidad del ámbito legal y el sistema de salud, se concluye que sería interesante realizar un abordaje empírico para dar cuenta con detalle cómo funcionan ambos espacios, pensados desde la teoría de los campos de Bourdieu.

Palabras claves: Médicos, abogados, capital, homología.

\begin{abstract}
The work aims to analyze theoretical reflection points from Bourdieu's perspective in the field of law and in the medical field.

Pierre Bourdieu, tangentially problematized the field of health, that is why we believe a contribution is necessary in the study of the health field.

On a conjectural plane, alien to empirical research, a greater number of analogies are detected than differences between the two social spaces. Perhaps, this situation finds an answer based on the unavoidable presence of the State, such as "metafield". Due to the historicity of the legal field and the health system, it is concluded that it would be interesting to carry out an empirical approach to give a detailed account of how both spaces work, thought from the theory of Bourdieu's fields.
\end{abstract}

Keywords: Doctors, lawyers, capital, homology.

\footnotetext{
- Maestrando en Sociología (CEA-UNC). Licenciado en Comunicación Social (FCC-UNC). Abogado (Facultad de Derecho, UNC). Ex Becario del Centro de Investigaciones Jurídicas y Sociales (Cijs-UNC).

diego_cevallos@hotmail.com
} 
Cevallos Ammiraglia, D.A. Campo jurídico y campo médico: algunas analogías y diferencias. Derecho y Ciencias Sociales. Noviembre 2020 - Abril 2021. No 24 .Pgs 1 -16 ISSN 1852-2971. Instituto de Cultura Jurídica y Maestría en Sociología Jurídica. Facultad de Ciencias Jurídicas y Sociales. Universidad Nacional de La Plata. Argentina.

\section{Campo jurídico y campo médico: algunas analogías y diferencias ${ }^{1}$}

Diego A. Cevallos Ammiraglia

\section{Introducción}

El trabajo adopta una perspectiva sociológica y tiene como objeto plantear algunas analogías y diferencias entre el campo jurídico (Bourdieu, 2000) y el campo médico. Ciertamente, Bourdieu, ha problematizado el campo del Derecho y tangencialmente el campo de la medicina. En su obra Homo Academicus cuando aborda el campo universitario, menciona semejanzas entre el campo médico y el jurídico, aunque al primero sólo lo aborda indirectamente.

Tanto la Medicina, como la Abogacía, son dos carreras que son consideradas "clásicas", sea por el caudal de personas que estudian ello, o porque tienen unos cuantos años de antigüedad en la cantera académica de las Universidades ${ }^{2}$. Comparten la legitimidad, la autorización social y científica. En esa clave, el artículo da algunas pistas para pensar ciertas similitudes o analogías, pero también diferencias entre estos dos campos.

Así, se esgrimen supuestos que deben ser tensionados con abordajes empíricos en situaciones concretas.

El artículo organiza las reflexiones en cuatro acápites. En el primer de ellos, a modo analítico, se conceptualiza la noción de campo de Bourdieu, y se presentan los rasgos estructurantes de los ámbitos jurídico y médico como espacio sociales. En segundo término, los habitus médico y jurídico son analizados. En tercer lugar, se aborda la violencia simbólica como dimensión presente y estructurante de ambos espacios. En cuarto y último lugar, se presentan algunas consideraciones finales, pero que no clausuran el estudio del campo médico y el campo jurídico como espacios sociales fuertemente análogos.

\section{Herramientas de Bourdieu para desentrañar el campo médico}

En este apartado, comenzamos dando cuenta de algunos aspectos centrales en la teoría de los campos de Bourdieu. Luego, nos centramos en el campo médico y el campo jurídico.

\footnotetext{
${ }^{1}$ Agradezco a la Dra. Julieta Capdevielle por contribuir de manera significativa a este artículo, recomendando lecturas posibles y también leyendo este texto en su versión embrionaria y en su faz final.

${ }^{2}$ Aun cuando el texto es teórico, cabe precisar que dichas afirmaciones son apoyadas en datos y bibliografía. Así, González et al (2011) sostienen que dos tercios de las universidades del país cuentan con la carrera de abogacía. Según lo enunciado por los autores, la carrera de abogacía tiene "la mayor matriculación anual en todo el país" (2011: 282). En relación al ámbito sanitario, hay que decir que en Argentina hay 47 universidades que tienen en su oferta académica a la carrera de medicina (Centeno y Campos, 2017). Centeno y Campos (2017) advierten que la medicina es la quinta carrera más elegida en el país, después de Administración, Abogacía, Economía y las Ingenierías. En relación a los orígenes de las unidades académicas en suelo argentino, cabe decir que en 1791 se creó la cátedra de la Instituta, que dio lugar a la Facultad de Jurisprudencia en Córdoba. A partir de 1883, la Universidad Nacional de Córdoba entrega el título de abogado. En relación a la medicina, en 1821 se crea la primera carrera de medicina del país, en la Universidad de Buenos Aires.
} 
Cevallos Ammiraglia, D.A. Campo jurídico y campo médico: algunas analogías y diferencias. Derecho y Ciencias Sociales. Noviembre 2020 - Abril 2021. No 24 .Pgs 1 -16 ISSN 1852-2971. Instituto de Cultura Jurídica y Maestría en Sociología Jurídica. Facultad de Ciencias Jurídicas y Sociales. Universidad Nacional de La Plata. Argentina.

Pierre Bourdieu, fue un autor que se interesó por el funcionamiento del poder en distintos ámbitos y cómo las desigualdades perduraban, entre otras cuestiones. En ese marco, abordó el campo político, el económico, el universitario, el burocrático, el jurídico, entre otros. No realizó un abordaje del campo médico, a pesar de que en Homo Academicus, da cuenta de algunos aspectos del funcionamiento de las Facultades de Medicina. Es por ello que se torna necesario abordar a priori algunos aspectos significativos y estructurantes del campo de la salud.

Antes de avanzar con el estudio de los espacios sociales en cuestión, cabe precisar que el concepto de campo, central en la teoría del sociólogo francés, supone un conjunto de posiciones y de relaciones entre posiciones, actuales y potenciales, jerárquicas, ocupadas por agentes que detentan un capital específico que está en juego en el campo y que por ello se lucha, por el monopolio del mismo. Como señalan Bourdieu y Wacquant (2005:150):

"En términos analíticos, un campo puede ser definido como una red o una configuración de relaciones objetivas entre posiciones. Estas posiciones están objetivamente definidas, en su existencia y en las determinaciones que imponen sobre sus ocupantes, agentes o instituciones, por su situación presente y potencial en la estructura de distribución de especies de poder (o capital) cuya posesión ordena el acceso a ventajas específicas que están en juego en el campo, así como por su relación objetiva entre posiciones (dominación, subordinación, homología, etc.)."

En esa concepción relacional y asentada sobre una matriz agonística, donde los agentes ingresan a jugar al campo porque hay un interés, una illusio, un reconocimiento sin cuestionamiento a las normas del juego, el concepto de "lucha" se constituye como fundamental. La teoría de Bourdieu es fuertemente relacional. Sus conceptos son una red, unos unidos con otros. En ese marco, el concepto de campo presupone el de capital. Aquí, hay un alejamiento de las teorías marxistas que conciben a éste último de manera solamente económica (Capdevielle, 2011). Bourdieu, se aleja de ello y utilizando una mirada "mercantil"3, sostiene que "el control diferencial de poderes - capitales- constituye el vector de definición de posiciones (Capdevielle y Freyre, 2013). El capital es la carta de ingreso al campo y el elemento por el que se lucha dentro de él. El capital es poder, "recurso crítico" (Capdevielle y Freyre, 2013:115) que se disputa en el campo.

Adentrándonos en los espacios sociales que nos interesan comparar, cabe precisar que

\footnotetext{
${ }^{3}$ En el sentido de que hace una metáfora entre el campo y el mercado.
} 
Cevallos Ammiraglia, D.A. Campo jurídico y campo médico: algunas analogías y diferencias. Derecho y Ciencias Sociales. Noviembre 2020 - Abril 2021. No 24 .Pgs 1 -16 ISSN 1852-2971. Instituto de Cultura Jurídica y Maestría en Sociología Jurídica. Facultad de Ciencias Jurídicas y Sociales. Universidad Nacional de La Plata. Argentina.

Bourdieu aportó elementos significativos para pensar el funcionamiento del campo del Derecho ${ }^{4}$. El campo jurídico, es un espacio social relativamente autónomo, al interior del cual se produce y se dispersa autoridad jurídica (García Inda, 2001), forma por antonomasia “de violencia simbólica legítima cuyo monopolio pertenece al Estado" (García Inda, 2001:38). La disputa en el campo jurídico 5 (Bourdieu, 2000) está dada por la detentación legítima y monopólica del capital jurídico.

El capital jurídico consiste en decir qué es lo que dice el Derecho. En otras palabras, en la capacidad técnica y socialmente reconocida de interpretar un corpus de textos que consagra la perspectiva legítima del universo social (Bourdieu, 2000: 160). El "significado" del texto normativo es objeto de contienda en el campo jurídico "entre distintas hermenéuticas ya que la lectura es una forma de apropiarse de la fuerza simbólica que se encuentra allí [encerrada] en estado potencial" (Ciuffolini, 2017:266).

Por el lado del campo médico, lo primero que cabe precisar es que, como en todo campo, el capital en juego es central para su definición y el de los agentes que juegan dentro del campo. Así, la mirada de Bourdieu, no es una mirada sustancialista, donde se prefijan de antemano qué agentes son los que luchan en el campo. Además, la construcción del objeto científico, es tarea intelectual del investigador, en tanto, lo contrario sería caer en un realismo ingenuo (Bourdieu, Passeron y Chamboredon, 2002).

El campo médico, ciertamente, podría denominarse "campo de la salud”. Esto es así, porque el capital en juego sería detentando por diversos agentes, que ciertamente no todos son médicos. En efecto, podría haber otros agentes que luchen en el campo, como son los enfermeros, nutricionistas, fisioterapeutas, etc. En efecto, el propio Pierre Bourdieu, en Homo Academicus, habla del "campo médico" aunque sus palabras desbordan la Medicina en sentido estricto. Desde la manera de nombrar, se pone de relieve la lucha que se da dentro del campo médico. Desde la nominación del campo, se pone de manifiesto cierta hegemonía de la mirada médica en el campo de la salud. Difícilmente, aunque posible, en términos empíricos pueda separarse para su indagación únicamente a las personas egresadas de la carrera de Medicina.

\footnotetext{
${ }^{4}$ Es preciso advertir que Bourdieu dedicó un texto que tituló "Elementos para una sociología del campo jurídico" y desde el cual se desprendieron múltiples fuentes secundarias ancladas en este texto que analiza el funcionamiento del campo del derecho (García Inda, 1997,2001; por ejemplo).

$5 \mathrm{Ni}$ bien comienza Bourdieu a plasmar sus aportes a la Sociología Jurídica, menciona quienes son sus interlocutores. Intelectualmente dialoga y discute con el formalismo jurídico y el "instrumentalismo". El formalismo, afirma la autonomía del derecho, como esfera separada completamente de otras dinámicas, como la social o la política. En cambio, el "instrumentalismo" concibe al Derecho como un instrumento al servicio de la clase dominante (Bourdieu, 2000). Es la idea marxista. Para no adoptar la ideología de la independencia del derecho, sin caer en la concepción instrumentalista, según Bourdieu (2000:158) es necesario tener en cuenta la existencia de "un universo social relativamente independiente en relación a las demandas externas en cuyo interior se produce y se ejerce la autoridad jurídica, forma por excelencia de la violencia simbólica legítima cuyo monopolio pertenece al Estado y que puede servirse del ejercicio de la fuerza física".
} 
Cevallos Ammiraglia, D.A. Campo jurídico y campo médico: algunas analogías y diferencias. Derecho y Ciencias Sociales. Noviembre 2020 - Abril 2021. No 24 .Pgs 1 -16 ISSN 1852-2971. Instituto de Cultura Jurídica y Maestría en Sociología Jurídica. Facultad de Ciencias Jurídicas y Sociales. Universidad Nacional de La Plata. Argentina.

En el campo médico, la autora Castro Vásquez (2011) sostiene que el capital específico en el campo sanitario, es el saber médico y la gestión de la información médica ${ }^{6}$. A decir verdad, si bien esto es importante, consideramos más atinada la idea de considerar que en el campo sanitario el capital por el cual se lucha sería la imposición de una cosmovisión dominante legítima en todo el campo (Castro y Erviti, 2015). Esto implicaría, modos de pensar el perfil profesional de las diversas profesiones que ocupan el campo, modos de actuar en la relación entre profesionales y para con las personas que buscan atención médica, formas institucionales de pensar y diagramar la política pública, y, por supuesto, mecanismos de articulación de las Facultades de Ciencias Médicas - central en el campo universitario- (Bourdieu, 2014) y los nosocomios formadores de profesionales.

El combate o lucha por el capital es una de las propiedades generales, comunes, transversales, de todos los campos (Bourdieu, 1990). En ambos tipos de capital específico, tanto jurídico como médico, se aprecia la presencia del Estado. La lógica estatal constructora del orden de lo simbólico y de espacios sociales, estaría impregnada en las palabras "sagradas" de los magistrados y los profesionales de la salud. Estos capitales, son objeto de lucha en sus respectivos campos, y encuentran en el Estado un "metacampo" (Bourdieu, 2014b).

En ambos campos, jurídico y médico, habría una división entre "profanos" y "profesionales" (Bourdieu, 2000). Habría un corte que nada tendría de contingente, de accidental o de impensado. Al contrario, existiría toda una logística de trabajo simbólico que haría funcionar al sistema con apariencia de independencia de las relaciones de fuerza (Bourdieu, 2000; García Inda, 2001).

Uno de los indicios de ello, está dado por el lenguaje. En el campo jurídico, el vocabulario es técnico, como en todo ámbito profesional, pero además es contraintuitivo, críptico, ensimismado, cerrado. No da una pizca de luz cuando se está desprovisto del habitus jurídico, propio de quién es profesional en el campo. Los verbos se conjugan en tercera persona y se utilizan formas impersonales. En el campo médico, mientras tanto, la "retórica de la autonomía" (Bourdieu, 2000) estaría dada por un lenguaje técnico fuertemente biologicista, cifrado (Menéndez, 1988 y Castro Vásquez, 2008), con agregados en idioma inglés y, a veces, impersonal.

En el espacio de la salud, los "pacientes"7 son usuarios del sistema de salud. No podríamos aseverar que "ingresan" al campo médico, al menos que detenten un mínimo de capital específico. De manera analógica, en el campo del derecho, el "profano" no ingresa al campo

\footnotetext{
${ }^{6}$ En un sentido similar, los autores Castro Pérez y Villanueva Lozano (2019) sostienen que el conocimiento sanitario es el capital médico en juego en el campo médico.

${ }^{7}$ Véase de la denominación utilizada, que denota un mundo simbólico distinto a "cliente".
} 
Cevallos Ammiraglia, D.A. Campo jurídico y campo médico: algunas analogías y diferencias. Derecho y Ciencias Sociales. Noviembre 2020 - Abril 2021. No 24 .Pgs 1 -16 ISSN 1852-2971. Instituto de Cultura Jurídica y Maestría en Sociología Jurídica. Facultad de Ciencias Jurídicas y Sociales. Universidad Nacional de La Plata. Argentina.

jurídico, lo que ingresa es su interés, su conflicto, que se transformará en un conflicto jurídico y en un diálogo entre expertos, configurándose así lo que Bourdieu (2000) denomina transmutación.

El abismo o frontera entre "profanos" y "profesionales" es uno de los fundamentos del funcionamiento y el mantenimiento de la institución "espacio judicial” o "espacio de salud humana" como ámbitos que funcionarían como neutros (Bourdieu, 2000). Esto supone que en el proceso de transmutación se produciría una neutralización ya que habría un "contrato" en donde el profano toma distanciamiento de su interés, el cual pasa a ser objeto de tratamiento entre expertos (Bourdieu, 2000). Así, el juez que interpreta o lee una sentencia o el médico que diagnostica un cuadro patológico, por ejemplo, están cubiertos por una suerte de reserva de autoridad, donde su discurso está investido de decisión legítima y oficial. En esa clave, el discurso médico modelará cuerpos. Dice Fernández Agis: “(...) la medicina pasará, en las sociedades modernas, a desempeñar funciones normativas (2015:308)." De esta manera, el discurso, sea jurídico o médico, crea lo que nombra. Consagra, legitima una institución instituida.

El campo jurídico y el campo médico, son creados por el Estado, que es una suerte de "metacampo" (Bourdieu, 2014b). El Estado monopoliza diversos capitales, crea subcampos. Es quien otorga los títulos, el capital cultural institucionalizado (Gutiérrez, 2005) quien delega en Colegios Profesionales el control de las matrículas profesionales. Además, uno de los Poderes que componen el Estado, es el Judicial. En esa clave, la distancia entre los "profanos" y los "profesionales" es aún más intensa y remarcada en ambos campos, jurídico y médico. Los "profesionales" de los campos en análisis, al igual que los agentes del campo burocrático, gozan de una retórica oficial y de una dramatización en diversos actos. La selección de jueces o el juramento hipocrático médico, podrían ser leídos en clave de dramatización "teatral” (Goffman, 1981) de un acto de eficacia simbólica ${ }^{8}$.

La comparación entre dos campos, no puede ser ajena a la idea de homología (Bourdieu, 2000, 2007 y Gutiérrez, 2002). Como sostiene Gutiérrez (2005), señalar rasgos estructurales análogos

\footnotetext{
${ }^{8}$ El juez, el abogado y quienes detentan capital jurídico, se expresan en decisiones judiciales o en escritos o alegatos orales. Estos discursos, supuestamente, expresan la voluntad de las normas jurídicas, no de la visión del juzgador ni del letrado. El campo jurídico, tiene como particularidad el despliegue de autoridad jurídica (García Inda, 2001). Bajo el ropaje y vestimenta del "interés común" los agentes del campo jurídico, emplean altas dosis de poder simbólico, bajo una suerte de "cinismo oficial” (García Inda, 2001), más aún si estos agentes pertenecen al campo burocrático. En esa lógica, un enfoque que se detenga en el desnivel entre profanos y profesionales, nunca puede obviar los vestigios simbólicos que refuerzan la autoridad y el poder (Bourdieu, 2000 y Rojo, 2005). Como ejemplos: el tamaño del despacho, la altura y material del sillón del juez, como así también, el acto de sentenciar en el marco de un juicio, entre otros. En el marco de la medicina, el discurso médico, vehículo de un saber específico, también despliega violencia simbólica y generalmente se realiza en situaciones donde se "teatraliza". En ese marco, los símbolos son fundantes, el uniforme, por ejemplo.
} 
Cevallos Ammiraglia, D.A. Campo jurídico y campo médico: algunas analogías y diferencias. Derecho y Ciencias Sociales. Noviembre 2020 - Abril 2021. No 24 .Pgs 1 -16 ISSN 1852-2971. Instituto de Cultura Jurídica y Maestría en Sociología Jurídica. Facultad de Ciencias Jurídicas y Sociales. Universidad Nacional de La Plata. Argentina.

en los distintos espacios sociales, permite analizar las propiedades comunes y específicas de los campos en comparación. El principio de la homología funcional y estructural (Gutiérrez, 2002 y 2005) supone que, en primer término, todos los campos se organizan con la misma lógica, una distribución desigual, inequitativa de los recursos críticos (Capdevielle y Freyre, 2013). En segundo lugar, el principio se sustenta en que las oposiciones entre los más ricos y los que menos detentan un capital específico son homólogas entre los campos y además, equivalentes a las posiciones que organizan el campo de las clases sociales (Gutiérrez, 2002 y 2005).

En el marco de este principio, en clave del primer fundamento sobre el cual se cimienta el principio de homología, como propiedad común a todos los campos, hay una distribución desigual del capital específico (Gutiérrez, 2002 y 2005). En el campo médico, a priori, podría haber una tendencia a que los agentes con mayor cantidad de capital son quienes ocupan posiciones directivas en Instituciones de la salud, por ejemplo. En igual sintonía, en el campo jurídico, a priori, también habría una tendencia a que los agentes con mayores cantidades de dosis de capital ocupen posiciones jerárquicas, por ejemplo, los jueces. Esta similitud, quizá, pueda tener asidero en el alto grado de institucionalización de ambos campos, creados por el propio Estado.

Conforme el segundo fundamento del principio de homología, las oposiciones equivalentes entre los "más ricos y menos ricos" (Gutiérrez, 2005: 59) que poseen un capital específico, pueden encontrarse en ambos espacios sociales analizados. Entre el campo médico y el jurídico, existe una correspondencia entre las posiciones más jerárquicas y las subordinadas. A priori, los agentes que tienen en sus arcas mayor cantidad de capital específico en juego, se ubican en posiciones jerárquicas, ya sea en el campo médico o en el jurídico. A manera meramente ejemplificativa, los funcionarios ministeriales de la salud podrían tener su equivalente en los funcionarios de la órbita administrativa en materia judicial. Mientras que los agentes con menos capital, se ubicarían en posiciones desventajosas. A modo ejemplificativo, pueden mencionarse a flamantes egresados que se incorporan al mercado laboral. Ahora bien, de manera coherente con nuestra mirada teórica, no solamente los egresados serían los que ocuparían posiciones desjerarquizadas en el campo (médico o jurídico). A priori, podría mencionarse la posición jerárquica del cuerpo de médicos con trayectoria en relación a otros profesionales del campo sanitario, también con trayecto profesional. Estos supuestos podrían ser tensionados con abordajes empíricos en situaciones concretas.

Asimismo, las posiciones homólogas nos revelan también las oposiciones que organizan la clase social, el nivel socio-económico (Gutiérrez, 2005). Quienes se encuentran en las altas esferas decisivas del campo médico, es decir, quienes ocupan posiciones más jerárquicas en el campo, $a$ 
Cevallos Ammiraglia, D.A. Campo jurídico y campo médico: algunas analogías y diferencias. Derecho y Ciencias Sociales. Noviembre 2020 - Abril 2021. No 24 .Pgs 1 -16 ISSN 1852-2971. Instituto de Cultura Jurídica y Maestría en Sociología Jurídica. Facultad de Ciencias Jurídicas y Sociales. Universidad Nacional de La Plata. Argentina.

priori, no pareciera que se encuentra en la misma posición que un practicante recién recibido u otros profesionales de la salud, aun cuando tengan trayectoria profesional. Ciertamente hay matices que aparecen y se evidenciarían en un abordaje empírico. Esto encuentra paralelo en el campo jurídico, por ejemplo, entre un juez y un abogado recién egresado u otros profesionales (aún con trayectoria) que disputan sentidos de una norma jurídica. En efecto, la teoría de Bourdieu no es sustancialista, no son los agentes los que definen el campo, sino que éste cobra forma a partir del capital en juego. Así, los ejemplos con agentes que tienen "nombre propio" son simplemente a los fines de ilustrar las premisas teóricas.

\section{La constitución disposicional: distancia y autoridad}

La relación entre el habitus y el campo es una relación de supeditación: el campo estructura el habitus, que es el fruto de las estructuras objetivas, de las condiciones objetivas (Gutiérrez, 2002 y 2005). Además, el habitus contribuye a formar el campo como mundo vivo, concediendo sentido y significado a las estructuras (Gutiérrez, 2002 y 2005). La realidad social existe dos veces, en las cosas y en los cuerpos, en los campos y en los habitus, en el exterior y en el interior de los agentes (Capdevielle, 2011, Gutiérrez, 2005). Siendo más claros, el habitus es:

“...un sistema de disposiciones duraderas y transferibles, estructura estructurante y estructurada, que funciona como un principio generador y ordenador de las prácticas y de representaciones que pueden estar objetivamente adaptadas a su fin sin suponerla búsqueda consciente de fines ni el dominio expreso de las operaciones necesarias para alcanzarlos, objetivamente 'regladas' y 'regulares' sin ser en nada el producto de la obediencia a reglas y, siendo todo esto, colectivamente orquestadas sin ser el producto de la acción organizadora de un director de orquesta.”(Bourdieu, 2007:86).

Así, el habitus es un esquema de disposiciones profundas, duraderas (ya que tienden a persistir el tiempo), que han sido construidas, estructuradas, por las condiciones objetivas, pero a su vez, son esquemas estructurantes porque engendran prácticas y pensamientos. A su vez, las prácticas sociales suponen estrategias que son "razonables" en tanto son explicadas a partir de las condiciones objetivas y de las propias disposiciones del agente (Gutiérrez, 2004 y 2005; Bourdieu, 2007; García Inda, 2001). Las prácticas sociales, y por ende las estrategias, desde esta perspectiva, no son el resultado de cálculos conscientes, aunque no por ello son irrazonables. 
Cevallos Ammiraglia, D.A. Campo jurídico y campo médico: algunas analogías y diferencias. Derecho y Ciencias Sociales. Noviembre 2020 - Abril 2021. No 24 .Pgs 1 -16 ISSN 1852-2971. Instituto de Cultura Jurídica y Maestría en Sociología Jurídica. Facultad de Ciencias Jurídicas y Sociales. Universidad Nacional de La Plata. Argentina.

Es prudente advertir que los rasgos de los habitus jurídicos y médicos aquí mencionados, no agotan la totalidad del abordaje. Más aún, sólo constituyen suposiciones y no conclusiones de abordajes empíricos propios.

En el campo jurídico como el campo médico, el habitus de los profesionales podría ser constituido por un sentido de la distancia neutralizante 9 (Bourdieu, 2000). Sería una especie de imperativo de función (Bourdieu, 2000) que estaría inscripto en lo más profundo de las disposiciones. Esta distancia en el trato humano y la diferencia en la conversión del espacio mental y de la postura lingüística (Bourdieu, 2000) puede ser leído en clave de estrategia de los agentes. No es una táctica “cínica” (Bourdieu, 1990 y García Inda, 2001), sino que la estrategia es devenida del sentido práctico con el que operan los agentes en los campos. Es una maestría (García Inda, 2001), una lógica práctica, no mentada, sino una economía de la práctica. Es producto del habitus, como condicionante del dominio simbólico de las prácticas (García Inda, 2001). En esa lógica, la distancia y el lenguaje, pueden ser leídas en clave de estrategias de los agentes.

Cuando estas estrategias suponen un trato docto entre los profesionales, léase en el saludo cordial amigable por título máximo obtenido, consagración del reconocimiento del capital cultural institucionalizado, que se hace cuerpo a la manera de disposiciones duraderas, profundas y curriculares, podemos advertir que estamos ante estrategias ortodoxas (Bourdieu, 2014 y 1990). Esta clase de estrategias, se ejecutan en búsqueda de mantener el status quo y el monopolio del capital. Otra estrategia que podría evidenciarse en un abordaje empírico, es la pretendida ocupación de parte de médicos y abogados de puestos institucionales que permitan moldear el cuerpo de doctos. Dice Bourdieu (2014:107): "El poder propiamente universitario está fundado principalmente en el dominio de los instrumentos de reproducción del cuerpo profesional, jurado de agregación, comité consultor de las universidades (...).” Si bien es cierto que se está refiriendo al campo universitario, allí Bourdieu expresa que se está refiriendo justamente a la Facultad de Ciencias Médicas y a la Facultad de Derecho.

\footnotetext{
${ }^{9}$ En el campo jurídico existe un desnivel marcado entre profanos y profesionales. Dentro del campo, existe un trabajo continuo de racionalización por parte de los trabajadores simbólicos (Bourdieu, 2000). Esta labor continua es realizada para que el sistema parezca totalmente independiente de las tensiones de las relaciones de fuerza. En ese sentido, cuando un conflicto es transmutado en conflicto jurídico, hay una "neutralización" de lo que está en juego en cada contienda, en tanto cuando el conflicto ingresa al campo jurídico, el profano renuncia a la violencia física y en cambio, acepta las reglas del campo. Entre ellas, podemos encontrar que el conflicto se resuelve en un dialogo entre expertos (Bourdieu, 2000 y García Inda, 1997). Así, el justiciable no puede convertir su postura lingüística y mental y deja en manos de agentes especializados el tratamiento de su conflicto, lo cual implica tomar distancia de su propio conflicto (Bourdieu, 2000). El ingreso del interés del justiciable al campo del derecho, es el "nutriente" que asegura la existencia del personal especializado y de su monopolio (Bourdieu, 2000). "Son los propios agentes del campo jurídico que producen la necesidad de sus propios servicios" (Bourdieu, 2000:191-192). Consideramos que en el campo de la salud también podría advertirse una "transmutación" del problema, en tanto el ojo del profesional convierte a la dolencia en una categoría médica. Así, el paciente queda subordinado a las sugerencias médicas.
} 
Cevallos Ammiraglia, D.A. Campo jurídico y campo médico: algunas analogías y diferencias. Derecho y Ciencias Sociales. Noviembre 2020 - Abril 2021. No 24 .Pgs 1 -16 ISSN 1852-2971. Instituto de Cultura Jurídica y Maestría en Sociología Jurídica. Facultad de Ciencias Jurídicas y Sociales. Universidad Nacional de La Plata. Argentina.

Si en el campo médico está en juego la imposición de una mirada dominante legítima, o en otras palabras, el principio de dominación de las dominaciones (Bourdieu, 2007), el habitus médico, es ese pack disposicional que podría inclinar al cuerpo profesional médico a querer ocupar posiciones que tengan dominación sobre otras posiciones de jerarquía. Un ejemplo podría consistir en los agentes médicos que regulan la práctica profesional de los estudiantes de Medicina en los Hospitales. En el campo jurídico, sucede algo similar. La admisión o no de determinados agentes a determinados puestos de trabajo o a cierta titulación podría ser un puesto estratégico que combina el poder propio del campo con poder institucional.

Por otro lado, podríamos advertir una dimensión en el habitus que atiende a la socialización profesional. Sellado en el habitus, las disposiciones tienden a hacer pensar y actuar a los agentes de manera determinada. En efecto, el habitus profesional es modelado sobre la base de las experiencias familiares primeras, situaciones en la vida estudiantil y en la práctica profesional. ${ }^{10}$ El campo jurídico podría ser considerado el campo del desnivel por antonomasia. Su composición y su funcionamiento así pacerían ponerlo de relieve. En el marco de la abogacía, bajo el modelo de enseñanza jurídica hegemónico (el positivismo jurídico), se aprehende un fuerte sentido de la jerarquía ${ }^{11}$ (Brígido et al, 2009 y Kennedy, 2000). No sólo el rango se pone de relieve en el trato humano, trato amigable por capital cultural institucionalizado, sino también y fundamentalmente en otras exteriorizaciones del habitus. En esa clave, la manera de hablar, de comportarse e incluso, de vestir, son aprehendidas e incorporadas con eficacia por el alumnado (Brígido et al, 2009). Así, el vestir una corbata, no es un detalle menor. Esta situación podría evidenciar un desnivel entre quien es profesional y quien es profano (Bourdieu, 2000) ${ }^{12}$.

El habitus jurídico se conforma con fuertes críticas a los contenidos aprendidos, aunque las disposiciones se solidifican y ratifican, por lo general, en lo que refiere a la identidad abogadil y a un modelo de "ser abogado" (Brígido et al, 2009). En esa lógica, así como en el campo de las creencias hay que tener creencias en el campo (Arriaga, 2013), en el campo de la legalidad hay que creer en la legalidad del campo. Se aceptan las reglas, hay interés en jugar en el campo y acumular el capital es la máxima.

\footnotetext{
${ }^{10}$ Aquí aparece la noción de habitus primarios y secundarios. Los primarios responden a las condiciones objetivas y experiencias primeras (familiares) mientras que los habitus secundarios son los que se construyen en el resto de nuestras experiencias (Capdevielle, 2011).

${ }^{11}$ No es objeto de este trabajo detallar los modelos epistémicos de la enseñanza del derecho, sin embargo, cabe advertir que el perfil profesional hegemónico que se moldea en la enseñanza jurídica (al menos en Córdoba, Argentina) es el abogado tradicional o liberal. Su relación con los "clientes" suele ser jerárquica, en tanto se ha socializado bajo fuertes pautas obediencias ante la autoridad. Mientras que las relaciones entre pares es escasa, la relación para con el docente es de subordinación del alumnado (Brígido et al, 2009).

${ }^{12}$ Estas suposiciones son de una parcialidad de los agentes del campo jurídico, ya que como mencionamos antes, la detentación del capital define al campo.
} 
Cevallos Ammiraglia, D.A. Campo jurídico y campo médico: algunas analogías y diferencias. Derecho y Ciencias Sociales. Noviembre 2020 - Abril 2021. No 24 .Pgs 1 -16 ISSN 1852-2971. Instituto de Cultura Jurídica y Maestría en Sociología Jurídica. Facultad de Ciencias Jurídicas y Sociales. Universidad Nacional de La Plata. Argentina.

El campo de la salud, presenta también una compleja red de relaciones, muchas de ellas, jerárquicas (Castro, 2014). El campo se compone de múltiples profesionales, no sólo médicos. Así, pareciera, a priori de un abordaje empírico, que las relaciones de autoridad y poder no sólo se marcan entre médicos y pacientes, sino entre profesiones distintas. Conjuntamente, sería interesante indagar las tensiones y relaciones entre médicos de distinta trayectoria y especialidad. Asimismo, en la profesión médica, generalmente, el cuerpo del paciente habla sin que el paciente abra su boca. Los síntomas están allí, en su rostro febril, en sus dolencias musculares, en sus signos exteriores que dialogan con el médico ${ }^{13}$. En el estudio jurídico, como una de las alternativas posibles de trabajo, el "cliente" es quien acude y cuenta su historia. En otras palabras, en el caso de los abogados (uno de los agentes del campo jurídico), el interés del cliente ingresa al campo del derecho y se produce lo que Bourdieu (2000) denomina transmutación. Hay un "contrato" de entrada, donde el justiciable ${ }^{14}$ acepta que su conflicto sea transformado en un conflicto jurídico y por ende, en un diálogo entre los agentes del campo jurídico (Bourdieu, 2000). En el campo de la salud, el paciente parecería quedar ajeno a las decisiones sobre su cuerpo. El profesional es quien tendría la providencia. Salvo algunas excepciones extremas, parecería que hay una situación similar al campo del derecho.

Otra analogía entre ambos espacios sociales, radicaría en que los agentes del campo médico al igual que los agentes del campo jurídico (sobre todo abogados), al margen de la institución donde hayan obtenido el capital cultural institucionalizado (Bourdieu, 1987), pueden desempeñarse en el ámbito privado o público. Tanto el habitus médico como el jurídico de los agentes podrían ser constituido con distintas lógicas, sean éstas más privatistas o más publicistas. No hay que soslayar la presencia de las lógicas privadas en ambos espacios sociales.

Una distinción significativa radica en el aprendizaje del "saber-hacer". Los educandos en profesiones de la salud, aprenderían haciendo. La asimilación en el habitus de formas de proceder, de pensar y actuar es contraída en el trabajo formativo diario, sea en las aulas universitarias o en los hospitales-escuela (Castro, 2014). Los agentes estudiantes, en las profesiones de la salud, egresan y sabrían hacer. Sabrían tomar la temperatura corporal, poner inyecciones, conocerían de medicamentos, entre otras cosas.

En cambio, en el campo jurídico, la tendencia pareciera ser otra. Se aprecia que la formación de los agentes que en mayoría luchan en el campo jurídico - puede haber otros que no hayan cursado estudios en Facultades de Derecho-, esto es, abogados y operadores jurídicos, es eminentemente

\footnotetext{
${ }^{13}$ Este trabajo se ubica en la perspectiva de Bourdieu, sin embargo, en un abordaje empírico podría ser fructífero producir dialogo entre las categorías de Pierre Bourdieu y las de Michel Foucault. Haciendo dialogar estos autores, el abordaje de la medicina como saber normalizador puede ser plausible. También, resulta loable emplear las categorías teóricas del paradigma biopolítico, iniciado por Foucault y continuado por diversos autores.

${ }^{14}$ Es el término utilizado en el campo del derecho para referir a "la parte" en el marco de un proceso judicial.
} 
Cevallos Ammiraglia, D.A. Campo jurídico y campo médico: algunas analogías y diferencias. Derecho y Ciencias Sociales. Noviembre 2020 - Abril 2021. No 24 .Pgs 1 -16 ISSN 1852-2971. Instituto de Cultura Jurídica y Maestría en Sociología Jurídica. Facultad de Ciencias Jurídicas y Sociales. Universidad Nacional de La Plata. Argentina.

teórica y carente de práctica. El saber-hacer podría ser adquirido en forma complementaria, en trabajos en estudios jurídicos o en otras instituciones. Como describe Begala (2006), la enseñanza jurídica adopta como modelo dominante la "clase magistral", donde el docente marca una asimetría con el alumnado y realiza diversas citas de textos legales, a su ritmo. No hay tiempo para detenerse a pensar situaciones prácticas ni tampoco para elaborar escritos judiciales. El saber-hacer, en consecuencia, es una deuda pendiente en los planes de estudio de Abogacía (Brígido, 2004). ${ }^{15}$

En definitiva, la forma de constitución del habitus en ambos campos posee una gran analogía. Es una mirada distante, asimétrica y autoritaria ${ }^{16}$ sobre "el otro". Intuimos que un trabajo empírico, meticuloso y cuidadoso, podría desentrañar los principales modos de constitución del habitus en profesiones de la salud y en los agentes dominantes del campo jurídico ${ }^{17}$.

\section{Acto clínico y aceptación del Derecho}

El acto clínico, es el acto por excelencia en el campo médico de ejercicio y despliegue de violencia simbólica ${ }^{18}$ (Castro, 2014; Bourdieu, 2014). La observación, en la práctica médica, se asienta en el ojo que va a normalizar y a distinguir una patología. Dice Bourdieu (2014:90): "La competencia clínica no puede funcionar en la práctica sino apoyándose en índices que le proporcionan los pacientes". En ese acto, cargado de rituales ${ }^{19}$, formas propias de la Medicina, institución que se instituye en las disposiciones más profundas de quienes asisten para obtener una respuesta médica, es en el cual se despliega mayores dosis de esto que Bourdieu denomina violencia simbólica ${ }^{20}(1996)$.

La violencia simbólica es sutilmente desplegada, aunque contundente en sus efectos. Sin una palabra de desperdicio, señala Bourdieu (2014:90): “Ese trabajo de producción de los síntomas que conduce al diagnóstico (...) se realiza en una relación asimétrica en la que el experto está en posición de imponer sus propios presupuestos cognitivos sobre los índices entregados por el

\footnotetext{
${ }^{15}$ Estos señalamientos se fundan en textos que dan cuenta de abordajes empíricos.

${ }^{16}$ Castro y Erviti (2015) sostienen que el habitus médico es autoritario, el cual se construye durante la formación como médico. Para el abordaje del campo médico, utilizan los aportes de Bourdieu y Foucault.

${ }^{17}$ Siempre con la advertencia de saber que los abogados no son los únicos y que el campo no está definido por los agentes, sino por el capital en juego.

${ }^{18}$ Para Castro y Erviti (2015) el campo médico posee una tensión clara entre un espacio para ejercicio de la ciudadanía y una estructura disciplinamiento. Entienden que las instituciones de salud ejercen control, vigilancia y disciplinamiento. En ese sentido, recurren a las categorías teóricas de Michel Foucault y las articulan con las de Bourdieu.

${ }^{19}$ La práctica médica supone entre otras "formas rituales": la emisión de turnos para la atención; el tiempo de espera convirtiendo a quien necesita la atención médica en "paciente"; la presencia de un espacio físico empleado para el área de secretaría que reserva turnos, cobra y controla el tránsito por la institución; la distribución de objetos en el consultorio (Ferrero, 2003); la exhibición de los títulos académicos; entre otros.

${ }^{20}$ Dicen Bourdieu y Passeron: (1996:42): "Todo poder de violencia simbólica, o sea, todo poder que logra imponer significaciones e imponerlas como legítimas disimulando las relaciones de fuerza en que se funda su propia fuerza, añade su fuerza propia, es decir, propiamente simbólica, a esas relaciones de fuerza".
} 
Cevallos Ammiraglia, D.A. Campo jurídico y campo médico: algunas analogías y diferencias. Derecho y Ciencias Sociales. Noviembre 2020 - Abril 2021. No 24 .Pgs 1 -16 ISSN 1852-2971. Instituto de Cultura Jurídica y Maestría en Sociología Jurídica. Facultad de Ciencias Jurídicas y Sociales. Universidad Nacional de La Plata. Argentina.

paciente (...)". Así, la violencia simbólica es una violencia ejercida con "complicidad" del dominado a la relación de dominación (Bourdieu, 2000). Es una violencia que se ejerce por aquiescencia del dominado al dispositivo de dominación. El dominado, no puede imaginar otra forma de ver el mundo, lo piensa en la misma clave construida desde la usina del dominante. El Modelo Médico Hegemónico (Menéndez, 1988) se inserta en la lógica del campo médico. Supone una mirada biologicista y ahistórica del cuerpo médico sobre las personas que buscan atención médica (Menéndez, 1988). Esto supondría que las personas que buscan atención sanitaria, podrían sólo pensar la salud en clave de asistencia por parte de profesionales médicos y no a partir del autocuidado, por ejemplo.

En la misma sintonía, el Derecho es aceptado porque las normas jurídicas son expresiones morfológicas por excelencia de eficacia simbólica (Bourdieu, 2000). La radiación de violencia "hipnótica" que suponen fundamenta la creencia en la ley, venerándola como justa sólo porque es ley (Bourdieu, 2000). En el campo jurídico se utiliza la universalización (Bourdieu, 2000) como factor significativo para que las personas acepten el Derecho. En el campo jurídico, la retórica de la autonomía, hace a su forma. En síntesis, la fuerza del derecho, está en su forma, no en su contenido (Bourdieu, 2000).

La fuerza de la violencia simbólica que irradia en el seno del campo del derecho, se pone de relieve por el hecho de que no sólo "copta" a los agentes del campo jurídico, que juega en el campo, sino que también logra complicidad por dominados denominados "profanos" (Bourdieu, 2000).

En sumatoria, en el seno de ambos campos, médico y jurídico, existe como supuesto estructurante el despliegue de autoridad y violencia simbólica. Esta situación permite el funcionamiento de ambos espacios con la apariencia de neutros ${ }^{21}$ y permite que el discurso jurídico y el alegato médico sean leídos por los "profanos" como veredictos oficiales y legítimos.

\section{Consideraciones finales}

\footnotetext{
${ }^{21}$ Ninguna práctica de los agentes es desinteresada. En esa lógica, lo específico, lo particular del campo jurídico "reside en una ilusión de neutralidad, de universalidad, de autonomía, de "desinterés" incluso" (García Inda, 2001: 40). El ámbito jurídico no es neutro, funciona como neutro. El trabajo de racionalización del derecho ejercido por los agentes del campo jurídico busca que las normas jurídicas aparezcan como autónomas de las relaciones de fuerza (Bourdieu, 2000). Esto hay que enmarcarlo en la teoría de los campos de Bourdieu, donde los campos poseen autonomía relativa. Además, el campo jurídico despliega violencia simbólica, la cual supone "complicidad" de los dominados, aquiescencia de quien sufre la violencia. Esta connivencia, en el campo jurídico, supone el reconocimiento del derecho y su contracara, el desconocimiento de su arbitrariedad (Bourdieu, 2000). En el campo médico, la situación sería análoga. Las prácticas no serían desinteresadas, por ende no habría neutralidad. La violencia simbólica desplegada "hipnotiza" a quienes la sufren y por ende, la apariencia de neutral surgiría como reconocimiento de una legitimidad al discurso médico.
} 
Cevallos Ammiraglia, D.A. Campo jurídico y campo médico: algunas analogías y diferencias. Derecho y Ciencias Sociales. Noviembre 2020 - Abril 2021. No 24 .Pgs 1 -16 ISSN 1852-2971. Instituto de Cultura Jurídica y Maestría en Sociología Jurídica. Facultad de Ciencias Jurídicas y Sociales. Universidad Nacional de La Plata. Argentina.

Analizar estos mundos, el campo médico y el jurídico, es analizar críticamente y científicamente dos espacios socialmente reconocidos y legítimos, basadas en operar una objetivación que aspira a la objetividad y a la universalidad (Bourdieu, 2014).

La develación de los resortes del poder y de "exotizar" lo doméstico, es un compromiso científico crítico, propio de la Sociología crítica, que aspira a realizar rupturas epistemológicas y a desnudar los principios de la acción que parecen camuflados en los aires cotidianos y en las dinámicas actorales diarias. Nada tiene de natural el comportamiento selectivo del selector, parafraseando a Bourdieu, cuando clasifica y elige una política, un agente para una práctica profesional o una forma de direccionar la dominación en campo.

En resumidas cuentas, se aprecian mayores analogías que diferencias, cuantitativamente hablando, entre el campo médico y el jurídico.

Siguiendo los apartados planteados de manera analítica, encontramos grandes analogías entre el campo médico y el jurídico. El capital específico, podría ser similar. En el campo jurídico, el capital en juego es el sentido legítimo del Derecho y a partir de ello la imposición de una cosmovisión, mientras que en el campo médico, el capital en juego supondría también la imposición de una forma de apreciar la diagramación del servicio de salud, un modo de concebir la enfermedad, los cuerpos, la salud, en síntesis, una cosmovisión que regla al campo de la salud.

En relación a estos dos campos, tenemos que destacar también que el Estado tiene una presencia ineludible. Como creador de campos, el Estado, a priori, parecería que insertara en la boca de los agentes estatales palabras oficiales y "sagradas". Además, entre ambos campos, a partir del principio de homología, se podrían aprecian particularidades y denominadores comunes.

En relación al habitus dominante, como historia hecha cuerpo, se aprecia, en ambos espacios sociales, la presencia de un agente moldeado en las agencias educativas, que ejerce su función profesional de manera distante y jerárquica.

En relación a la violencia simbólica, si bien son ejercidas de distinta manera, ambos espacios sociales comparten la fuerte presencia de este tipo de violencia, en el sentido de Bourdieu.

Finalmente, cabe aseverar que ambos espacios sociales pueden ser objeto de indagación empírica en aras de develar, en casos concretos, similitudes y diferencias. El texto, simplemente, se erige en un estímulo que podría permitir abrir interrogantes.

\section{Bibliografía}

Arriaga, G. (2013). El campo de la creencia y la creencia en el campo: Contribuciones para una sociología de las religiones en la obra de Pierre Bourdieu. (Trabajo Final de Grado). 
Cevallos Ammiraglia, D.A. Campo jurídico y campo médico: algunas analogías y diferencias. Derecho y Ciencias Sociales. Noviembre 2020 - Abril 2021. No 24 .Pgs 1 -16 ISSN 1852-2971. Instituto de Cultura Jurídica y Maestría en Sociología Jurídica. Facultad de Ciencias Jurídicas y Sociales. Universidad Nacional de La Plata. Argentina.

Universidad Nacional de La Plata. Facultad de Humanidades y Ciencias de la Educación, La Plata: Argentina.

Begala, S. (2006). Tensiones entre la racionalidad instrumental y la orientada por valores en el discurso regulativo de la enseñanza jurídica. Anuario IX. Córdoba: Centro de Investigaciones Jurídicas y Sociales. Pp. 527-547.

Bourdieu, P. (2014). Homo Academicus. México: Siglo XXI Editores.

Bourdieu, P. (2014b). Sobre o Estado. Curso no Collége de France (1989-1992). España: Anagrama.

Bourdieu, P. (2000). "Elementos para una sociología del campo jurídico". En Pierre Bourdieu. Gunther Teubner La fuerza del derecho. Bogotá: Siglo del Hombre y Universidad de los Andes.

Bourdieu, P. (2003). Los juristas, guardianes de la hipocresía colectiva. Jueces para la democracia. 47,3-5.

Bourdieu, P. (2007). El sentido práctico. Buenos Aires: Siglo XXI Editores.

Bourdieu, P. (1990). Algunas propiedades de los campos. En Sociología y Cultura, Bourdieu. México: Grijalbo.

Bourdieu, P. (1987). Los tres estados del capital cultural. Sociológica. 2,5.

Bourdieu, P y Passeron, J. (1996). La Reproducción. Elementos para una teoría del sistema de enseñanza. México: Fontamara.

Bourdieu, P., y Wacquant, L. (2005). Una invitación a la sociología reflexiva. Buenos Aires: Siglo XXI Editores.

Bourdieu,P. Passeron, J., y Chamboredon, J. (2002). El oficio del sociólogo. Buenos Aires: Siglo XXI Editores.

Brígido, A. (2004). Los abogados en ejercicio de la profesión y su perspectiva sobre la formación profesional. V Congreso Nacional de Sociología Jurídica. La Pampa.

Brígido, A., Begala, S., Lista, C., Tessio Conca, A. (2009). En La Socialización de los estudiantes de abogacía. Crónica de una metamorfosis. Córdoba: Hispania Editorial.

Capdevielle, J., y Freyre, L. (2013). El concepto de Lucha en la sociología de Bourdieu. Revista de Ciencias Sociales. 140, 111-124.

Capdevielle, J. (2011). El concepto de habitus: "Con Bourdieu y contra Bourdieu". Anduli. Revista Andaluza de Ciencias Sociales. 10, 31-45.

Castro, R. (2014). Génesis y práctica del habitus médico autoritario en México. Revista mexicana de Sociología. 76,2, 167-197.

Castro, R., y Erviti, J. (2015). Sociología de la práctica médica autoritaria: violencia obstétrica, anticoncepción inducida y derechos reproductivos. (Tesis Doctoral). Centro Regional de Investigaciones Multidisciplinarias. Universidad Nacional Autónoma de México. México.

Castro Vásquez, M. (2008). De pacientes a exigentes. Un estudio sociológico sobre la calidad de atención, derechos y ciudadanía en salud. Región y sociedad. 22,48, 283-290.

Castro Vásquez, M. (2011). Habitus lingüístico y derecho a la información en el campo médico. Revista Mexicana de Sociología. Universidad Autónoma de México. Vol.73, n²2. 
Cevallos Ammiraglia, D.A. Campo jurídico y campo médico: algunas analogías y diferencias. Derecho y Ciencias Sociales. Noviembre 2020 - Abril 2021. No 24 .Pgs 1 -16 ISSN 1852-2971. Instituto de Cultura Jurídica y Maestría en Sociología Jurídica. Facultad de Ciencias Jurídicas y Sociales. Universidad Nacional de La Plata. Argentina.

Castro Pérez, R., y Villanueva Lozano, M. (2019). El campo médico en México. Hacia el análisis de sus subcampos y sus luchas desde el estructuralismo genético de Bourdieu. Sociología. 34,97, 73-113.

Centeno, A., y Campos, S. (2017). La educación médica en Argentina. FEM. Revista de la Fundación Educación Médica. 20, 6, 265-271.

Ciuffolini, A. (2017). Análisis culturales del Derecho. En Lariguet (eds.) Metodología de la Investigación Jurídica Córdoba: Editorial Brujas. (pp. 265-272).

Fernández Agis, D. (2015). La mirada médica. Revisitando la interpretación de Michel Foucault. Anales médicos. Universidad de La Laguna. 60(4), 306-310.

Ferrero, L. (2003). Tiempo y ritual en la organización del cuidado médico. Cuadernos de Antropología Social. 18, 165-183.

García Inda, A. (1997). La violencia de las formas jurídicas. La sociología del poder y el derecho en Pierre Bourdieu. Barcelona: Cedes. (Primera edición).

García Inda, A. (2001). “Introducción. La Razón del Derecho: entre habitus y campo". En Bourdieu, Poder, Derecho y Clases Sociales. Bilbao: Descleé de Brouwer. Pp. 9-50.

Goffman, E. (1981). La presentación de la persona en la vida cotidiana. Buenos Aires: Amorrortu Editores.

González, M., Marano, M.., Lista, C., Bianco, C., y Carrera, C. (2011). La formación de los abogados y la educación jurídica en Argentina. En González y Lista (comp.) Sociología Jurídica en Argentina: Tendencias y perspectivas. Buenos Aires: Eudeba. pp. 279-334.

Gutiérrez, A. (2004). Poder, habitus y representaciones: recorrido por el concepto de violencia simbólica en Pierre Bourdieu. Revista Complutense de educación. 15,1, 289-300.

Gutiérrez, A. (2002). Acerca de campo y habitus como categoría analíticas. Páginas. Revista de la Escuela de Ciencias de la Educación. $\mathrm{N}^{\circ} 2$ y 3. Disponible en https://revistas.unc.edu.ar

Gutiérrez, A. (2005).Las prácticas sociales: una introducción a Pierre Bourdieu. Córdoba: Ferreyra Editor.

Kennedy, D. (1990). La educación legal como preparación para la jerarquía. Desde otra mirada. Buenos Aires: Eudeba.

Menéndez, E. (1988). Modelo Médico Hegemónico y Atención Primaria. Segundas Jornadas de Atención Primaria de la Salud. Buenos Aires.

Rojo, R. (2005). Por una Sociología Jurídica, del Poder y la Dominación. Sociologias. Sociedade e Direito. Revista del Instituto de Filosofía e Ciencias Humanas. 6,13, 33-81. 